\title{
The Scottish Centre for Macromolecular Imaging - Evaluation of the JEOL CryoARM 300 and Direct Electron DE64 combination for automated cryoEM in a national cryo-EM centre.
}

David Bhella ${ }^{1}$ and James Streetley ${ }^{2}$

${ }^{1}$ MRC - University of Glasgow Centre for Virus Research, Glasgow, Scotland, United Kingdom, ${ }^{2}$ Scottish Centre for Macromolecular Imaging, University of Glasgow, United Kingdom

Cryo-transmission electron microscopy has undergone a technological revolution in recent years. It is now possible to achieve structural data on macromolecular assemblies such as proteins and nucleoprotein complexes at atomic and near-atomic resolutions. This has led to widespread interest in adopting the technology and significant investment in high-performance cryo-microscopes to serve structural biology communities. The Scottish Centre for Macromolecular Imaging (SCMI) was established by a consortium of Scottish Universities to provide access to cutting-edge cryo-EM resources for Scottish structural biologists. SCMI is a hub for a network of like-minded investigators providing training and support for the adoption of cryo-EM.

Cryo-EM instrumentation is most widely bought from two market-dominant companies - Thermo-Fisher (formerly FEI/Phillips) and Gatan. An important dimension of the SCMI project was our decision to purchase new to market instruments - The JEOL CryoARM automated $300 \mathrm{keV}$ cryo-microscope and a Direct Electron DE64 detector. In my talk, I will introduce SCMI and discuss our reasons for selecting these new tools over the market-dominant instrumentation. I shall outline the many challenges of being early-adopters and our progression through installation to service delivery.

With the CryoARM platform, JEOL brought several technical innovations to the field. Previously unstable and difficult to use, JEOL have reintroduced the cold FEG emitter as an easily managed bright, coherent electron source having a narrower energy spread than thermally assisted FEGs. Alongside a redesigned in-column energy filter, a newly designed autoloader and a supported installation of SerialEM, the CryoARM is an appropriate platform for automated data delivery in a national centre setting. I will present our analysis of CryoARM performance highlighting some of the benefits and challenges of working with this instrument.

The Direct Electron DE64 is an extremely versatile detector. The large format (8k x 8k) sensor, when used unbinned in either linear or electron counting mode allows capture of very large fields of view for tomography or single particle analysis of large objects. Used with binning and on-chip correlated double sampling, electron counting images may be recorded with excellent detective quantum efficiency.

My talk will cover the benefits and difficulties we have encountered when using the CryoARM 300/DE64 platform and present both single particle and tomography data generated at SCMI.

\section{References}

1. Bhella, Cryo-electron microscopy: an introduction to the technique, and considerations when working to establish a national facility. Biophys Rev (2019), doi:10.1007/s12551-019-00571-w. 